\title{
Structural and functional studies of the Thermus thermophilus 16S rRNA methyltransferase RsmG
}

\author{
STEVEN T. GREGORY, ${ }^{1,3}$ HASAN DEMIRCI, ${ }^{1,3}$ RICCARDO BELARDINELLI, ${ }^{1,2,3}$ TANAKARN MONSHUPANEE, ${ }^{1}$ \\ CLAUDIO GUALERZI, ${ }^{2}$ ALBERT E. DAHLBERG, ${ }^{1}$ and GERWALD JOGL ${ }^{1}$ \\ ${ }^{1}$ Department of Molecular Biology, Cell Biology and Biochemistry, Brown University, Providence, Rhode Island 02912, USA \\ ${ }^{2}$ Laboratory of Genetics, Department of Biology Molecular Cell Biology and Animal Biology, University of Camerino, 62032 \\ Camerino (MC), Italy
}

\begin{abstract}
The RsmG methyltransferase is responsible for $N^{7}$ methylation of G527 of 16S rRNA in bacteria. Here, we report the identification of the Thermus thermophilus rsmG gene, the isolation of rsmG mutants, and the solution of RsmG X-ray crystal structures at up to $1.5 \AA$ resolution. Like their counterparts in other species, T. thermophilus rsmG mutants are weakly resistant to the aminoglycoside antibiotic streptomycin. Growth competition experiments indicate a physiological cost to loss of RsmG activity, consistent with the conservation of the modification site in the decoding region of the ribosome. In contrast to Escherichia coli RsmG, which has been reported to recognize only intact 30S subunits, T. thermophilus RsmG shows no in vitro methylation activity against native $30 \mathrm{~S}$ subunits, only low activity with $30 \mathrm{~S}$ subunits at low magnesium concentration, and maximum activity with deproteinized 16S rRNA. Cofactor-bound crystal structures of RsmG reveal a positively charged surface area remote from the active site that binds an adenosine monophosphate molecule. We conclude that an early assembly intermediate is the most likely candidate for the biological substrate of RsmG.
\end{abstract}

Keywords: methyltransferase; $16 \mathrm{~S}$ rRNA; 7-methylguanine; S-adenosyl-L-methionine; ribosome modification

\section{INTRODUCTION}

The RNA components of the translational machinery are post-transcriptionally modified in all three domains of life. While tRNA modifications have been found to play significant roles in determining decoding specificity (Gustilo et al. 2008) and conformational stability of tRNA molecules (for instance, see Kinghorn et al. 2002; Shigi et al. 2006), functional roles for rRNA modifications have not been clearly established. The location of the majority of modifications in and around the decoding and peptidyltransferase active sites of the ribosome has been interpreted to imply an important role for modifications in ribosome function (Decatur and Fournier 2002; Chow et al. 2007). In particular, methylations concentrated in the decoding site of the 30 S ribosomal subunit may act to fine tune codon

\footnotetext{
${ }^{3}$ These authors contributed equally to this work.

Reprint requests to: Gerwald Jogl, Department of Molecular Biology, Cell Biology and Biochemistry, Brown University, Box G-E129, Providence, RI 02912, USA; e-mail: Gerwald_Jogl@brown.edu; fax: (401) 8636114.

Article published online ahead of print. Article and publication date are at http://www.rnajournal.org/cgi/doi/10.1261/rna.1652709.
}

recognition, in a manner similar to tRNA modifications. The modifications that have received the greatest attention are those that impact the sensitivity of ribosomes to the action of antibiotic inhibitors of protein synthesis. In addition to methyltransferase genes acquired by horizontal transfer are endogenous methyltransferase genes that are responsible for highly or universally conserved methylations and whose loss modifies antibiotic sensitivity. The best-known examples of these are $k s g A$ (Helser et al. 1972), tlyA (Johansen et al. 2006), and the recently identified $r s m G$ (Okamoto et al. 2007).

Originally designated as gidB, $r s m G$ encodes a methyltransferase responsible for the synthesis of $\mathrm{m}^{7} \mathrm{G} 527$ in $16 \mathrm{~S}$ rRNA (Escherichia coli numbering). The recognition that rsm $G$ encodes a methyltransferase resulted from the solution of the $E$. coli enzyme structure, which indicated a methyltransferase fold (Romanowski et al. 2002). More recently, rsmG mutants of E. coli (Okamoto et al. 2007), Streptomyces coelicolor (Nishimura et al. 2007a), and Bacillus subtilis (Nishimura et al. 2007b) were shown to lack the conserved 7-methylguanine modification at G527 of $16 \mathrm{~S}$ rRNA. These same studies found $r s m G$ mutants of these and several other bacterial species, including Staphylococcus 
aureus, Mycobacterium smegmatis, and Mycobacterium tuberculosis, to be weakly resistant to the aminoglycoside antibiotic streptomycin, which binds to the 30S subunit and makes contacts with the backbone of the 16S rRNA residues C526 and G527 (Carter et al. 2000). It was also found that $r s m G$ mutants accelerated the appearance of high-level streptomycin-resistance mutations in $\operatorname{rps} L$, encoding ribosomal protein $\mathrm{S} 12$, for reasons that remain obscure (Nishimura et al. 2007b; Okamoto et al. 2007). In support of the notion that $16 \mathrm{~S}$ rRNA modifications impact decoding accuracy, ribosomes from a $r s m G$ mutant of $B$. subtilis were found to be weakly hyper-accurate (Nishimura et al. 2007b), as are ribosomes carrying amino acid substitutions in ribosomal protein $\mathrm{S} 12$, encoded by $r p s L$ (for review, see Kurland et al. 1996).

Here we report the identification of the $\operatorname{rsm} G$ locus and rsmG mutants of the thermophilic bacterium Thermus thermophilus HB8, an analysis of the substrate specificity of RsmG, and the determination of the X-ray crystal structures of $T$. thermophilus RsmG complexed with cofactor $S$-adenosyl-L-methionine (AdoMet) and adenosine monophosphate bound to a putative rRNA binding site. T. thermophilus rsmG mutants, like those of other species, display a weak streptomycin-resistance phenotype. In contrast to the reported specificity of E. coli RsmG for intact 30 S subunits as substrate (Okamoto et al. 2007), we find that the T. thermophilus enzyme shows a marked preference for deproteinized 16S rRNA as substrate and is completely inactive with native $30 \mathrm{~S}$ subunits as substrate. Finally, we observed that T. thermophilus RsmG has unusual structural features: a C-terminal disulfide bond and an N-terminal covalent circularization.

\section{RESULTS}

\section{Identification of $T$. thermophilus rsmG, isolation of spontaneous rsm $G$ mutants, and construction of a $\Delta$ rsmG deletion strain}

The T. thermophilus HB8 locus TTHA1971, annotated as gidB in the unpublished genome sequence (GenBank accession number AP00826), encodes a protein that aligns well with other RsmG sequences. Consistent with the presence of an RsmG ortholog is the recent experimental demonstration of the presence of $\mathrm{m}^{7} \mathrm{G} 527$ in T. thermophilus HB8 16S rRNA (Guymon et al. 2006). To confirm the identity of TTHA1971 as $r s m G$, we identified three mutant alleles of $r s m G$, which had arisen in previous selections for streptomycin resistance (Gregory et al. 2001, 2009; Carr et al. 2005; Gregory and Dahlberg 2009), but could not be shown to carry mutations in either rpsL, encoding ribosomal protein $\mathrm{S} 12$, or in $\operatorname{rrs} B$, encoding $16 \mathrm{~S}$ rRNA.

The three $r s m G$ alleles are diagrammed in Figure $1 \mathrm{~A}$ and listed in Table 1. One mutant was found to have an insertion of the recently discovered IS element ISTth7, which exists in multiple copies in the HB8 genome (Gregory and Dahlberg 2008). The $r s m G::$ ISTth7 insertion generates a direct repeat from the codon for R221 to the codon for V224, alters the C-terminal 26 amino acids, and extends RsmG by 214 amino acids. Another allele, $r s m G 1$, is a -1 frameshift mutation resulting from deletion of one of eight consecutive Cs spanning codons for T232, P233, and P234. This alters the C-terminal 15 amino acids and extends RsmG by 107 amino acids. A third allele, rsmG2, is $\mathrm{a}+1$ frameshift mutation resulting from insertion of a single C in a run of six C's spanning the codons for A193, P194, and L195. Because the open reading frames for rsmG and the immediately downstream parA overlap and are out of frame by one nucleotide, the $r s m G 2+1$ frameshift mutation alters amino acids 195-249 and creates an inframe RsmG-ParA fusion protein. This RsmG-ParA fusion does not produce any obvious growth defect, which is surprising given that ParA is involved in chromosome segregation during cell division. However, N-terminal GFP-ParA fusion proteins localize normally in B. subtilis (Murray and Errington 2008), and B. subtilis parA-null alleles do not exhibit deleterious phenotypes (Ireton et al. 1994). All three of the T. thermophilus rsmG alterations presumably produce streptomycin resistance by interfering with the methyltransferase activity of RsmG by extending the $\mathrm{C}$ terminus or altering critical C-terminal residues. These results strongly suggest that TTHA1971 is, in fact, rsmG.

To more conclusively demonstrate the direct relationship between $\operatorname{rsm} G$ and the $\mathrm{m}^{7} \mathrm{G} 527$ modification and to provide a source of unmodified $30 \mathrm{~S}$ ribosomal subunits to use as in vitro substrates for RsmG, we constructed an $r s m G$ null allele by deleting the $r s m G$ coding sequence and replacing it with $h t k$, encoding a heat-stable kanamycin adenyltransferase (Hashimoto et al. 2001). This null allele retains the very $\mathrm{N}$ - and C-terminal rsm $\mathrm{G}$ coding sequences, in-frame with the $h t k$ coding sequence, in order to maintain the $r s m G-$ parA overlap and minimize any effects on parA expression. This allele was designated $\Delta r s m G:: h t k 2$ and the mutant containing this allele was designated HG 917.

\section{Streptomycin-resistance phenotype of rsmG mutants}

The spontaneous $r s m G$ mutants and the $\Delta r s m G:: h t k 2$ null mutant were assessed for levels of resistance to streptomycin. Previous studies have shown that such mutants confer low-level streptomycin resistance (Nishimura et al. 2007a,b; Okamoto et al. 2007), and our rsmG1 and rsmG2 frameshift mutants and the ISTth7 insertion mutant were originally isolated on this antibiotic. Overnight cultures of wild-type T. thermophilus HB8 and the $\operatorname{rsm} G$ frameshift and insertion mutants, as well as the $\Delta r s m G:: h t k 2$ null mutant, were streaked for single colonies on Thermus enhanced medium (TEM) plates or TEM plates containing streptomycin sulfate at 25,50 , or $100 \mu \mathrm{g} / \mathrm{mL}$. A highly resistant $r p s L$ mutant containing the K87R substitution in ribosomal protein S12 


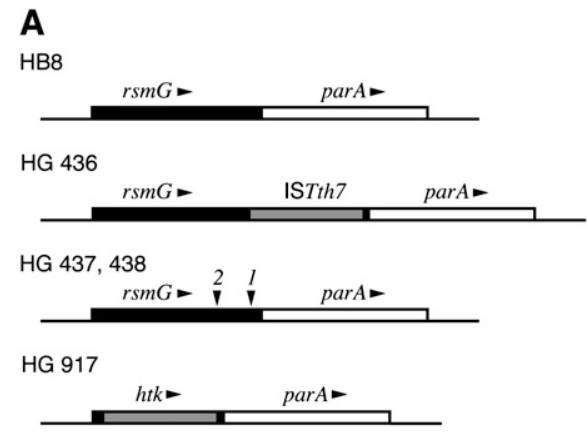

B
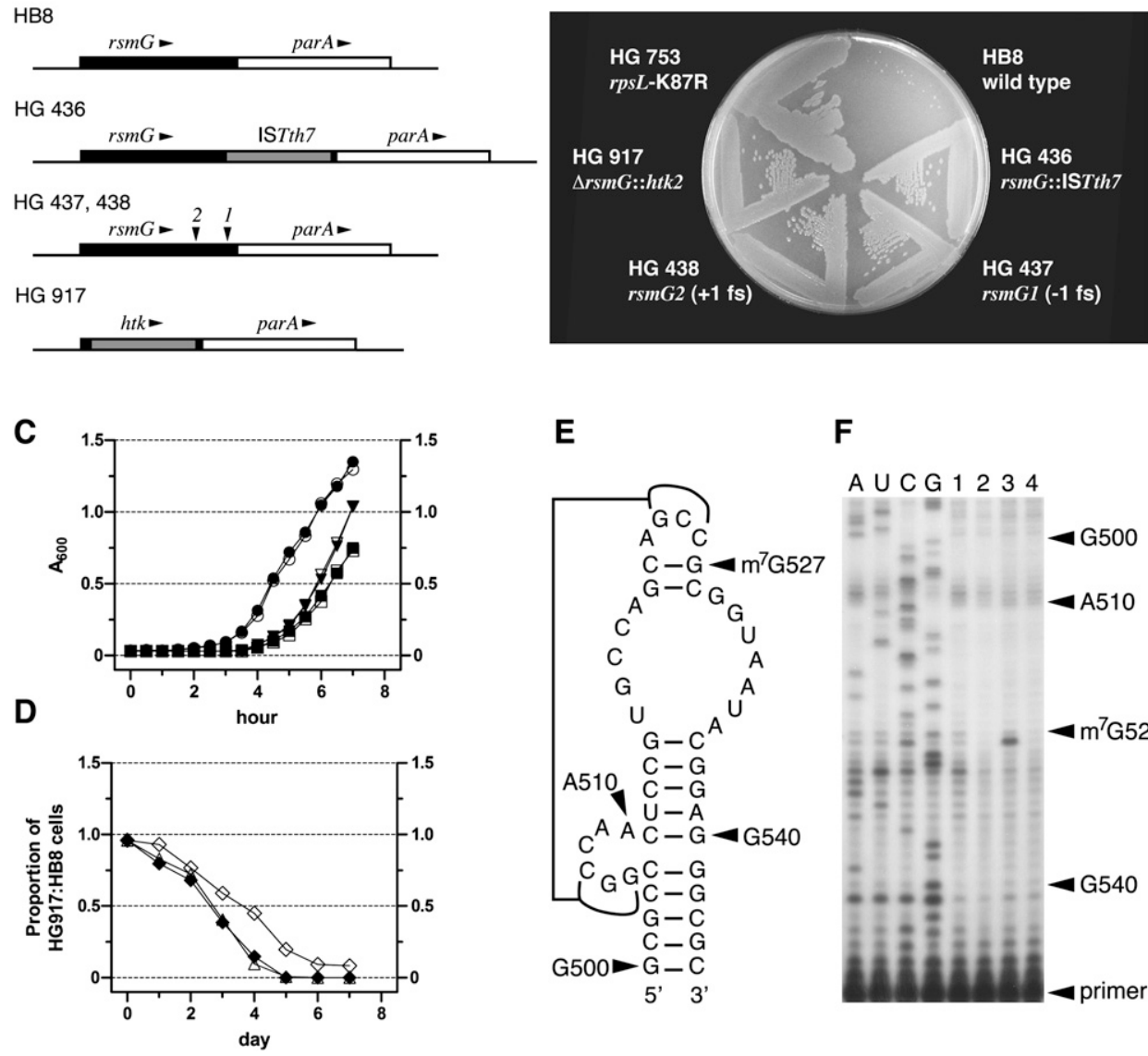

E

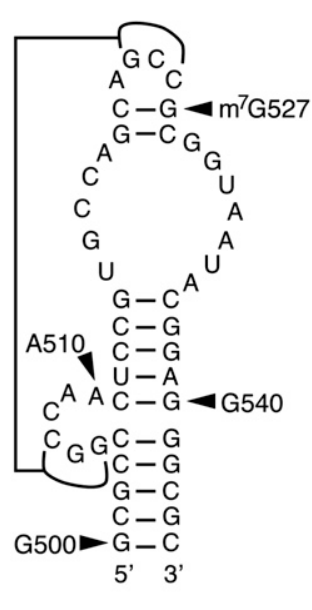

$\mathbf{F}$

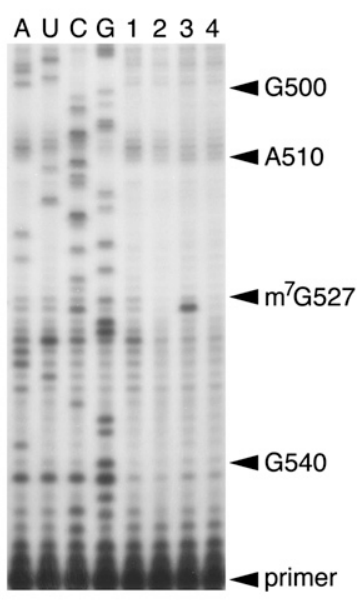

FIGURE 1. (A) Mutant alleles of $r s m G$ identified or constructed in this study. The location of the insertion sequence ISTth7 in HG 436 and the organization of the $\Delta r s m G:$ : htk2 allele in HG 917 are indicated. For HG 437 and HG 438, vertical arrowheads indicate the positions of the rsmG1 and $r s m G 2$ frameshift mutations (represented by italicized numerals 1 and 2, respectively). (B) Streptomycin-resistance phenotypes of the $r s m G$ alleles. HG 753 carries the K87R amino acid substitution in ribosomal protein S12 and is highly streptomycin-resistant. ( $C$ ) Growth of wild-type HB8 (filled symbols) and HG 917 (open symbols) at $60^{\circ} \mathrm{C}$ (inverted triangles), 72 (circles), and $80^{\circ} \mathrm{C}$ (squares). Data represent the means of three independent experiments, with a maximum standard deviation of 0.15 . (D) Growth competition between HB8 and HG 917 . The ratio of HG 917 cells to $\mathrm{HB} 8$ cells cocultured at $60^{\circ} \mathrm{C}$ (open diamonds), $72^{\circ} \mathrm{C}$ (filled diamonds), and $80^{\circ} \mathrm{C}$ (triangles). Data represent the means of three independent experiments with a maximum standard deviation of 0.14 . (E) Secondary structure of helix 18 of T. thermophilus $16 \mathrm{~S}$ rRNA showing the site of the $\mathrm{m}^{7} \mathrm{G} 527$ modification (left). (F) Primer extension analysis of $16 \mathrm{~S}$ rRNA from wild-type T. thermophilus HB8 and from the $\Delta r s m G:: h t k 2$ deletion strain HG 917; A, U, C, G, dideoxy sequencing lanes. Lane 1, wild-type HB8, untreated; lane 2, HG 917 untreated; lane 3, wild-type $\mathrm{HB} 8$, treated with $\mathrm{NaBH}_{4}$ and aniline; and lane 4, $\mathrm{HG} 917$, treated with $\mathrm{NaBH}_{4}$ and aniline. Arrowheads mark the locations of $\mathrm{m}^{7} \mathrm{G} 527$ and several landmarks for orientation.

was included for comparison. As shown in Figure 1B, all rsm $G$ mutants were resistant to $25 \mu \mathrm{g} / \mathrm{mL}$ streptomycin, including the $\Delta r s m G:: h t k 2$ null mutant, which had not previously been exposed to streptomycin. The $\operatorname{rsm} G$ mutants are all partially inhibited at $50 \mu \mathrm{g} / \mathrm{mL}$ streptomycin and severely inhibited by $100 \mu \mathrm{g} / \mathrm{mL}$ (data not shown). In contrast, the rpsL-K87R mutation confers resistance up to $1000 \mu \mathrm{g} / \mathrm{mL}$ (Gregory et al. 2001). Since all $r s m G$ mutants behaved identically, the $\Delta r s m G:: h t k 2$ null mutant HG 917 was used in all subsequent experiments.

To assess the effects of $r s m G$ deficiency on growth of $T$. thermophilus, we established growth curves of wild-type $\mathrm{HB} 8$ and $\mathrm{HG} 917$ at $60^{\circ} \mathrm{C}, 72^{\circ} \mathrm{C}$, and $80^{\circ} \mathrm{C}$ (Fig. 1C). At no temperature was a difference in growth rate for the two strains detected. However, in growth competition experiments (Fig. 1D) at all three temperatures, HG 917 was eventually outgrown by the wild-type strain, indicating a physiological cost of $\mathrm{m}^{7} \mathrm{G} 527$ deficiency.

\section{Primer extension analysis of $\mathrm{m}^{7} \mathrm{G527}$}

To assess the modification status of G527 in our rsmG null mutant, we performed primer extension analysis of $16 \mathrm{~S}$ rRNA extracted from 70S ribosomes. G527 is immediately adjacent to bases participating in a pseudoknot structure in 16S rRNA helix 18 (Fig. 1E). Methylation of the N7 of guanosine residues in RNA imparts on the glycosidic bond susceptibility to cleavage with aniline after reduction of the 
TABLE 1. Bacterial strains used in this study

\begin{tabular}{|c|c|c|c|c|}
\hline Strain & Genotype & Phenotype & Sm resistance ${ }^{a}$ & Source \\
\hline HB8 & Wild type & - & $<25 \mu \mathrm{g} / \mathrm{mL}$ & ATCC 27634 \\
\hline HG 436 & $\Delta r r s A:: h t k 1$ & $\mathrm{SmR}$ & $100 \mu \mathrm{g} / \mathrm{mL}$ & Spontaneous SmR mutant (Gregory and Dahlberg 2008) \\
\hline HG 436 & rsmG::ISTth7 & KanR & $100 \mu \mathrm{g} / \mathrm{mL}$ & Spontaneous SmR mutant (Gregory and Dahlberg 2008) \\
\hline HG 437 & rsmG1 (-1 frameshift) & $\mathrm{SmR}$ & $100 \mu \mathrm{g} / \mathrm{mL}$ & Spontaneous SmR mutant (this study) \\
\hline HG 438 & rsmG2 (+1 frameshift) & SmR & $100 \mu \mathrm{g} / \mathrm{mL}$ & Spontaneous SmR mutant (this study) \\
\hline HG 917 & $\Delta r s m G:: h t k 2$ & SmR & $100 \mu \mathrm{g} / \mathrm{mL}$ & Gene replacement (this study) \\
\hline HG 917 & $\Delta r s m G:: h t k 2$ & KanR & $100 \mu \mathrm{g} / \mathrm{mL}$ & Gene replacement (this study) \\
\hline HG 421 & $r p s L-K 87 R$ & SmR & $1000 \mu \mathrm{g} / \mathrm{mL}$ & $\begin{array}{l}\text { Spontaneous SmR mutant of } T \text {. thermophilus IB-21 } \\
\text { (Gregory et al. 2001) }\end{array}$ \\
\hline HG 753 & $r p s L-K 87 R$ & SmR & $1000 \mu \mathrm{g} / \mathrm{mL}$ & $\begin{array}{l}\text { Transformation of } T \text {. thermophilus HB8 } \\
\text { with DNA from HG } 421\end{array}$ \\
\hline
\end{tabular}

${ }^{a}$ Values represent the concentration of streptomycin sufficient to severely or completely inhibit growth.

N7-C8 bond with $\mathrm{NaBH}_{4}$ (Peattie 1979; Zueva et al. 1985). This cleavage is detectable by primer extension with reverse transcriptase (RT). As indicated in Figure 1F, a strong RT stop is observed at the position immediately preceding G527 with rRNA from wild-type T. thermophilus HB8 (Fig. 1F, lane 3), but not with rRNA from the $\Delta r s m G:: h t k 2$ knockout strain HG 917 (Fig. 1F, lane 4). This result further supports the conclusion that TTHA1971 is equivalent to $\operatorname{rsm} G$ and indicates that $\operatorname{rsm} G$ is the sole locus responsible for the production of $\mathrm{m}^{7} \mathrm{G} 527$ in $T$. thermophilus HB8.

\section{Methylation of $30 \mathrm{~S}$ ribosomal subunits and deproteinized $16 S$ rRNA by recombinant RsmG}

We next examined the substrate preference for T. thermophilus RsmG in vitro, using as substrate either wild-type $30 \mathrm{~S}$ subunits or $30 \mathrm{~S}$ subunits from the $\Delta$ rsmG::htk2 null mutant HG 917. We performed these experiments at both high $(10 \mathrm{mM}) \mathrm{Mg}^{2+}$ and low $(1 \mathrm{mM}) \mathrm{Mg}^{2+}$ ion concentrations, as we had previously observed increased activity at sub-physiological $\mathrm{Mg}^{2+}$ concentrations for the rRNA methyltransferase KsgA (Demirci et al. 2009). In vivo methylated 30 S subunits from wild-type T. thermophilus HB8 are, as expected, completely inactive as substrates at both $10 \mathrm{mM}$ and $1 \mathrm{mM} \mathrm{Mg}^{2+}$ (Fig. 2A). Surprisingly, subunits from the rsmG null mutant were also inactive at $10 \mathrm{mM} \mathrm{Mg}^{2+}$, but showed substantial activity $(\sim 10 \%$ completion after 90 $\min$ ) at $1 \mathrm{mM} \mathrm{Mg}^{2+}$. This result suggests that $30 \mathrm{~S}$ subunits in their native conformation are not a proper substrate and that removal of $\mathrm{Mg}^{2+}$ ions from the subunit is required to open the structure sufficiently to expose elements involved in enzyme binding.

Given the relatively low extent of reaction with $30 \mathrm{~S}$ subunits even at low $\mathrm{Mg}^{2+}$, we next examined the efficiency of methylation of $16 \mathrm{~S}$ rRNA deproteinized by phenol and chloroform extraction (see Materials and Methods). RsmG shows no activity with $16 \mathrm{~S}$ rRNA from wild-type T. thermophilus at either 10 or $1 \mathrm{mM} \mathrm{Mg}^{2+}$ (Fig. 2B). The reactions at 10 and at $1 \mathrm{mM} \mathrm{Mg}^{2+}$ with $16 \mathrm{~S}$ rRNA from the rsm $G$ knockout strain is much more efficient (to $75 \%$ of completion) and are also much faster (the reader will note the difference in time scales of the reactions). These results exclude the possibility of an effect of $\mathrm{Mg}^{2+}$ concentration on enzyme activity as the explanation for our observations with intact 30 S subunits and indicate that the most active substrate for T. thermophilus RsmG in vitro is deproteinized $16 \mathrm{~S}$ rRNA. These observations stand in contrast to those

A

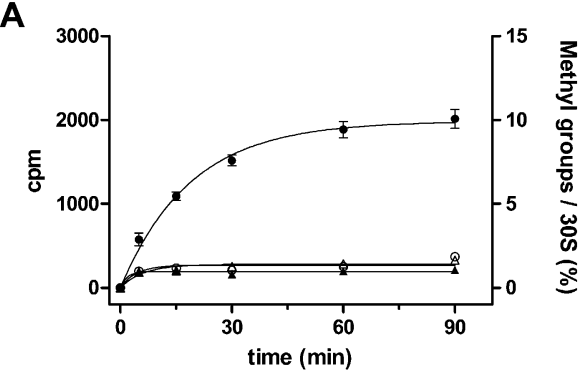

B

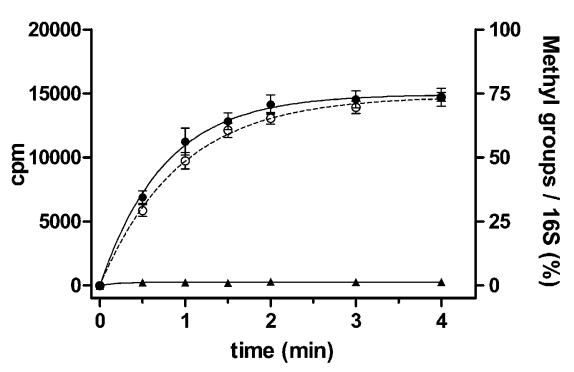

FIGURE 2. In vitro methylation of $30 \mathrm{~S}$ ribosomal subunits and $16 \mathrm{~S}$ rRNA by RsmG. (A) Methylation of intact $30 \mathrm{~S}$ subunits from Tth $\mathrm{HB} 8$ at $10 \mathrm{mM} \mathrm{MgOAc}_{2}$ (filled triangles) and $1 \mathrm{mM} \mathrm{MgOAc}_{2}$ (open triangles); methylation of $30 \mathrm{~S}$ subunits from $\mathrm{HG} 917$ at $10 \mathrm{mM}$ $\mathrm{MgOAc}_{2}$ (open circles) and $1 \mathrm{mM} \mathrm{MgOAc}$ (filled circles). (B) Methylation of $16 \mathrm{~S}$ rRNA from $\mathrm{HB} 8$ (filled triangles) and $\mathrm{HG} 917$ at $1 \mathrm{mM}$ $\mathrm{MgOAc}_{2}$ (filled circles) and at $10 \mathrm{mM} \mathrm{MgOAc}$ (open circles). Curves have been fitted with a single term equation and represent a duplicate experiment. Values are means \pm SEM. Note the difference in time scales for the two sets of data. 
from an earlier report indicating that E. coli RsmG preferentially methylates intact $30 \mathrm{~S}$ subunits and does not methylate deproteinized 16S rRNA (Okamoto et al. 2007).

\section{Crystal structure determination of RsmG}

We have determined the structure of RsmG (249 amino acids) in three different crystal forms. We report four RsmG data sets, three with cofactor AdoMet and one with AdoHcy (Table 2). One of the RsmG-AdoMet complexes also contained adenosine monophosphate (AMP), identifying a putative RNA-binding site. The structure was initially solved for data set RsmG1 in space group $\mathrm{P} 6_{1}$ by molecular replacement with a Modeller (Marti-Renom et al. 2000) homology model derived from the B. subtilis RsmG structure (PDB code 1XDZ; Midwest Center for Structural Genomics). The refined model from this data set was then used as a search model for molecular replacement of the two other crystal forms in data sets RsmG3 and RsmG4. The crystallographic $R / R_{\text {free }}$ factors are $0.17 / 0.20$, $0.18 / 0.21,0.17 / 0.23$, and $0.18 / 0.20$ for the four data sets, respectively. The majority of residues $(96.6 \%)$ are in the most favored region of the Ramachandran plot for all data sets, and there are no residues in the disallowed region.
Electron density for the final models were well defined in data sets RsmG1-3 except for residues in a highly conserved loop region (residues 46-57) near the active site (Fig. 3; discussed below). This conserved loop region is stabilized by crystal contacts and therefore well defined in data set RsmG4. Unlike data sets RsmG1-3, the first eight $\mathrm{N}$-terminal residues are disordered in data set RsmG4 and not included in the final model. The data collection and refinement statistics are given in Table 2.

The overall structure of RsmG contains a canonical class I methyltransferase catalytic domain with a central sevenstranded $\beta$-sheet that is flanked on both sides by three helices of varying length (Fig. 4B). This core domain is enlarged by the two $\mathrm{N}$-terminal helices $\alpha 1$ and $\alpha 2$ on one side and by helix $\alpha 9$ on the other side of the $\beta$-sheet. The overall structure remains highly similar in the different crystal forms with RMSDs for $\mathrm{C}_{\alpha}$ atoms of $0.33 \AA$, $0.29 \AA$, and $0.47 \AA$ between the structure in data set RsmG1 and the other three data sets, respectively. In the hexagonal crystal forms of data sets RsmG1-3, the first eight residues form an extended loop that is stabilized by crystal contacts and hydrogen bonds between Gly4 and Glu12 in helix $\alpha 1$.

The crystal structure of T. thermophilus RsmG indicates the existence of covalent modifications at the $\mathrm{N}$ and $\mathrm{C}$

TABLE 2. Data collection and refinement statistics

\begin{tabular}{|c|c|c|c|c|}
\hline & RsmG1 & RsmG2 & RsmG3 & RsmG4 \\
\hline Data collection & AdoMet & AdoMet, AMP & AdoHcy & AdoMet \\
\hline Space group & $\mathrm{P} 6_{1}$ & $\mathrm{P} 6_{1}$ & $\mathrm{P} 6_{1}$ & 1222 \\
\hline \multicolumn{5}{|l|}{ Cell dimensions } \\
\hline$a, b, c(\AA)$ & $77.8,77.8,167.7$ & $77.2,77.2,167.8$ & $134.8,134.8,167.1$ & $110.1,114.0,132.7$ \\
\hline$\alpha, \beta, \gamma\left({ }^{\circ}\right)$ & $90,90,120$ & $90,90,120$ & $90,90,120$ & $90,90,90$ \\
\hline Resolution $(\AA ̊)$ & $25-1.87(1.94-1.87)$ & $30-1.5(1.55-1.50)$ & $25-2.1(2.18-2.1)$ & $30-2.1(2.18-2.10)$ \\
\hline$R_{\text {merge }}$ & $0.089(0.49)$ & $0.12(0.38)$ & $0.099(0.49)$ & $0.063(0.53)$ \\
\hline$|/ \sigma|$ & $16.3(2.4)$ & $22.0(2.16)$ & $15.2(3.14)$ & $24.0(3.6)$ \\
\hline Completeness (\%) & $99.8(98.5)$ & $93.8(99.4)$ & $98.9(99.8)$ & $97.9(99.2)$ \\
\hline Redundancy & $4.5(4.2)$ & $6.7(3.4)$ & $4.9(4.8)$ & $5.6(5.6)$ \\
\hline \multicolumn{5}{|l|}{ Refinement } \\
\hline Resolution $(\AA)$ & $1.87(1.91-1.87)$ & $1.50(1.52-1.50)$ & $2.10(2.12-2.10)$ & $2.1(2.14-2.1)$ \\
\hline Number of reflections & $47,224(2575)$ & $84,494(2830)$ & $98,820(3137)$ & $47,884 / 2671$ \\
\hline$R_{\text {work }} / R_{\text {free }}$ & $0.17 / 0.20(0.24 / 0.30)$ & $0.18 / 0.21(0.22 / 0.25)$ & $0.17 / 0.23(0.21 / 0.28)$ & $0.18 / 0.20(0.24 / 0.29)$ \\
\hline \multicolumn{5}{|l|}{ Number of atoms } \\
\hline Protein & 3668 & 3722 & 11070 & 3700 \\
\hline Ligand & 54 & 54 & 156 & 54 \\
\hline Other & 21 & 46 & & 5 \\
\hline Water & 713 & 1026 & 1347 & 528 \\
\hline \multicolumn{5}{|l|}{$B$-factors } \\
\hline Protein & 26.61 & 22.43 & 36.97 & 38.04 \\
\hline Ligand & 21.14 & 15.52 & 50.31 & 26.1 \\
\hline Other & 44.26 & 35.87 & & 95.56 \\
\hline Water & 44.98 & 42.71 & 50.95 & 51.54 \\
\hline \multicolumn{5}{|l|}{ RMSD } \\
\hline Bond lengths $(\AA)$ & 0.008 & 0.02 & 0.006 & 0.003 \\
\hline Bond angles $\left(^{\circ}\right)$ & 1.48 & 2.22 & 1.32 & 0.78 \\
\hline
\end{tabular}

Note: One crystal is used for each data set. The highest resolution shell is shown in parentheses. 
FIGURE 3. Structure-based sequence alignment of RsmG homologs from T. thermophilus, B. subtilis, and E. coli. The position of secondary structure elements in the T. thermophilus structure is indicated. The position of the disordered region is indicated with a red box; residues in the cofactor-binding site are indicated with green boxes.

termini that are not found in two mesophilic RsmG structures. In all three data sets, we observed an additional electron density peak at covalent bonding distance between the $\mathrm{N}$-terminal $\alpha$-amino group and the side chain of His3. Analysis of bond distances and the crystallographic temperature factors after refinement indicates that this additional peak is most likely a carbon atom that is covalently bonded to the $\mathrm{N}$-terminal amino nitrogen atom and a nitrogen atom from the His3 imidazole side chain (Fig. 4B, inset). To our knowledge, such a cyclic linkage has not been observed previously. The most likely origin of this modification would be an intramolecular condensation reaction between an N-terminal formyl-group and the histidine imidazole nitrogen. We have observed this $\mathrm{N}$-terminal closure in several independent data sets and in two different crystal forms. Interestingly, we also observed a disulfide bond linking the RsmG C terminus between the penultimate Cys249 and Cys164, such that covalent linkages stabilize both termini from this molecule. Both features seem to be unique to the T. thermophilus enzyme based on sequence alignments with related enzymes (Fig. 3; Supplemental Fig. S1). While we cannot exclude the possibility that the N-terminal linkage is introduced into the recombinant protein upon expression in E. coli, there is accumulating evidence for the presence of disulfide bonds in intracellular proteins of thermophilic archaea and bacteria (Mallick et al. 2002; Beeby et al. 2005).

\section{Cofactor binding in the active site of RsmG}

As observed in other class I methyltransferases, AdoMet is bound in a canonical conformation above the $\beta$-sheet and close to the conserved GxGxG methyltransferase signature motif (residues 88-92 between strand $\beta 1$ and helix $\alpha 4$ ). The adenine ring is coordinated on one side in a mainly hydrophobic pocket that is lined by residues Leu39, Val110, and Val160 and by hydrogen bonds to the Ala139 main chain and to Glu140 on the other side. Both ribose hydroxyl groups form hydrogen bonds to Asp111, and the Lys116 side chain forms an additional hydrogen bond to the ribose $\mathrm{O} 3$ hydroxyl group. Arg158 interacts with the cofactor methionine carboxyl group, and the cofactor amino group is coordinated by interactions with mainchain carbonyl groups from Gly88, Phe93, and Arg158. The cofactor-binding site is accessible from the solvent region, and several well-defined solvent water molecules are observed forming hydrogen bonds to the cofactor.

The AdoMet cofactor is tightly bound in RsmG and copurifies with the recombinant protein. There was some indication of a loss of bound cofactor in data set RsmG3. This data set contains six molecules in the asymmetric unit. The cofactor electron density in all six molecules clearly showed that only AdoHcy was bound in this crystal form (the RsmG3 crystal form was obtained after crystallization periods of 2-3 mo). Occupancy refinement indicated that the cofactor-binding site is not fully occupied in this crystal form. In addition to these differences, we found that residues 159 to 161 in the cofactor-binding site assume two alternate conformations among the six molecules in this crystal form (Fig. 4E). In one of these orientations, Val160 participates in hydrophobic interactions with Leu166, Leu170, Leu87, and the AdoHcy adenine base. Val160 adopts a similar orientation in the other three data sets. In the second orientation, Val160 is oriented in the 


\section{A}

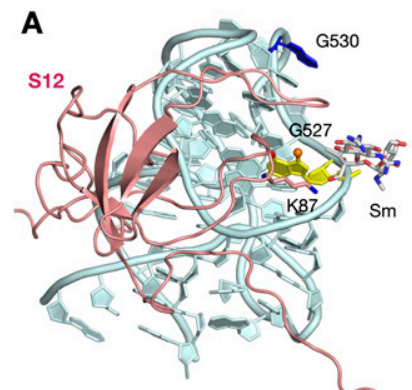

B

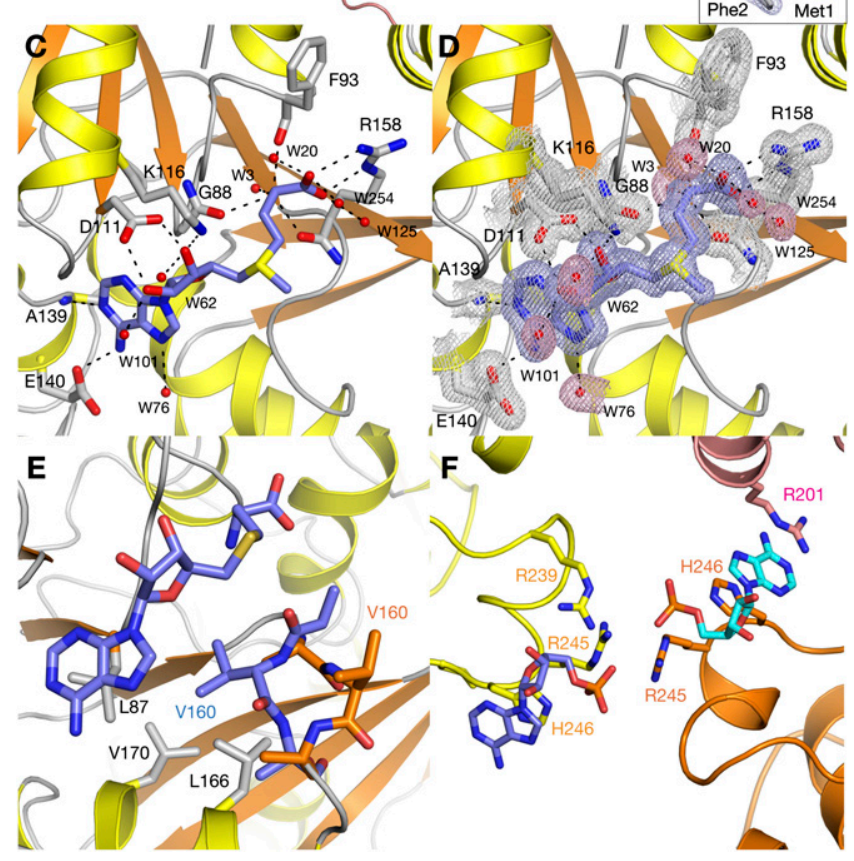

FIGURE 4. (A) Position of G527 as observed in the structure of helix 18 from the T. thermophilus $30 \mathrm{~S}$ subunit crystal structure (PDB 1FJG) (Carter et al. 2000). The N7 of G527 is shown as a sphere, and streptomycin is shown as sticks. The nearby, functionally critical residue G530 is colored blue. Ribosomal protein S12 (salmon) residue K87 is shown as sticks. (B) Schematic representation of the overall structure of RsmG. Secondary structure elements are colored in orange and yellow. AdoMet is shown in blue sticks, and the bound AMP molecule is shown as cyan sticks. $(C)$ Cofactor-binding site in RsmG. $(D)$ Final $\sigma_{\mathrm{A}}$-weighted $2 F_{\mathrm{O}}-F_{\mathrm{C}}$ electron density map of the cofactor-binding site contoured at the $1 \sigma$ level. $(E)$ Two alternate conformations observed for residues 159 to 161 in data set RsmG3. $(F)$ Crystal contacts mediated by the bound AMP molecule in data set RsmG2.

opposite direction and projects into the solvent region. This alternate conformation is observed only in data set RsmG3 and may represent a structural rearrangement of Val160 in response to cofactor binding.

To identify potential rRNA interacting surfaces in RsmG, we performed cocrystallization experiments using a mixture of nucleotides and nucleosides as crystallization additives. These experiments were prompted by our recent observation that guanosine binds in the active site of the RsmC methyltransferase (Demirci et al. 2008a). We observed significantly faster crystallization and improved crystal quality in the presence of adenosine monophosphate (AMP, data set RsmG2) when compared to the original crystallization conditions (data set RsmG1). In the RsmG2 structure, AMP is bound remote from the active site and engages in crystal contacts that include two other RsmG molecules and a second AMP molecule (Fig. 4F). The position of AMP marks this site as a potential RNA-binding surface.

\section{DISCUSSION}

\section{Structural comparison with related enzymes}

A database search with the Dali algorithm (Holm et al. 2008) confirmed that T. thermophilus RsmG is most closely related to the two available RsmG structures in the protein database (both annotated as GidB). The overall structure superimposes with the RsmG apo-enzyme structure from B. subtilis (PDB 1XDZ; Midwest Center for Structural Genomics) with an RMSD of $1.2 \AA$ for $213 \mathrm{C}_{\alpha}$ atoms. While the T. thermophilus $\mathrm{N}$ terminus, including helix $\alpha 1$, is considerably longer than that of the B. subtilis enzyme, the structure of the C-terminal region is well conserved between the two enzymes (Fig. 3). Key residues in the cofactor-binding site are also conserved. Val149 of $B$. subtilis RsmG (equivalent to Val160 in the mobile loop region of T. thermophilus RsmG) is oriented in an outward position similar to the outward loop conformation that is only observed in data set RsmG3. The similarity of this loop region in the B. subtilis apo-enzyme structure suggests that an analogous loop rearrangement upon cofactor binding may occur in this enzyme.

A second conserved loop, ${ }^{48} \mathrm{GKVNLTAL}^{55}$, was disordered in data sets RsmG1, RsmG2, and RsmG3 and not modeled in these three structures. Interestingly, this loop was also disordered in the E. coli RsmG structure. However, due to crystal contacts, this loop was ordered in data set RsmG4, and a defined conformation was also found in the B. subtilis RsmG structure. Nishimura et al. (2007b) reported that mutation of Thr42 (Thr53 in T. thermophilus) to asparagine in this highly conserved loop gives rise to streptomycin resistance, suggesting that the identity of this threonine residue and structural flexibility of the loop region are important for function. Flexible active site loops have also been observed in ribosomal protein L11 methyltransferase PrmA (Demirci et al. 2007, 2008b) and in the rRNA methyltransferase KsgA (Demirci et al. 2009).

As expected, T. thermophilus RsmG also superimposes well with E. coli RsmG (PDB 1JSX) (Romanowski et al. 2002) with an RMSD of $1.4 \AA$ for $167 \mathrm{C}_{\alpha}$ atoms. However, the E. coli apo-enzyme structure is considerably smaller (Figs. 3, 5A). The T. thermophilus $\mathrm{N}$-terminal extension is not conserved, and the E. coli $\mathrm{C}$ terminus does not extend beyond the last $\beta$-strand ( $\beta 7)$. The $C$-terminal loop region including helix $\alpha 10$ is not conserved between the two enzymes. However, this loop region provides the 


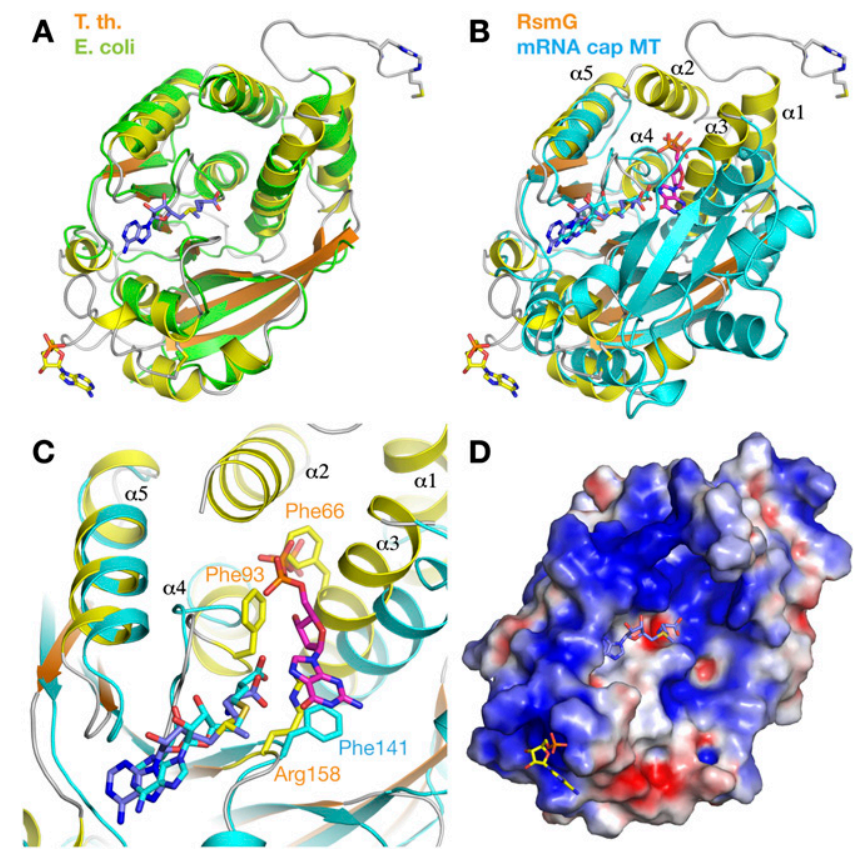

FIGURE 5. (A) Differences between the overall structures of RsmG from T. thermophilus (orange/yellow) and E. coli (green). AdoMet and AMP bound in the T. thermophilus enzyme are shown with blue and yellow sticks. $(B)$ Structural differences between RsmG (orange/ yellow) and the mRNA cap $\mathrm{m}^{7} \mathrm{G}$ methyltransferase (cyan). AdoHcy bound in the cap enzyme is shown in blue sticks, and the substrate guanine is shown in magenta sticks $(C)$ Comparison of the active-site region of RsmG with the cap methyltransferase colored as in $B$. $(D)$ Electrostatic surface representation $( \pm 8 \mathrm{KT} / \mathrm{e})$ of RsmG.

AMP-binding site in the T. thermophilus structure and may indicate a different mode of substrate interaction between the two enzymes. This may, in fact, partly explain the observed differences in substrate preference for the T. thermophilus and E. coli enzymes, as indicated earlier (see also below). A comparison of the active site region confirms that key residues are conserved between both enzymes. However, Arg139 (Arg158 in T. thermophilus) projects into the cofactor-binding pocket, and Arg123 (Arg138) is oriented into the solvent region in the E. coli apo-enzyme structure. In the mobile loop region, Ala140 replaces Val160 (seen to adopt two alternate conformations), and Phe141 replaces Ala161 and participates in hydrophobic interactions similar to that engaged by Val160 in the $T$. thermophilus structure.

In addition to RsmG homologs, the Dali search revealed that the RsmG catalytic domain shares significant homology with the mRNA cap $\mathrm{m}^{7} \mathrm{G}$ methyltransferase from Enzephalitozoon cuniculi (PDB 1RI1) (Fabrega et al. 2004). A comparison of the two enzymes, however, shows that the structural similarity is limited to the catalytic domains and the cofactor-binding site (Fig. 5B). In the cap enzyme structure, the guanosine triphosphate portion of the $\mathrm{m}^{7} \mathrm{GpppG}$ cap analog was observed bound in the active site. However, the guanosine cap analog does not serve as a useful indicator of the position of G527 in RsmG. The guanine base in the cap methyltransferase is located in a cleft between the catalytic domain and an additional smaller domain that is not present in RsmG. Furthermore, positioning the guanosine in RsmG in this same orientation would create a clash between the phosphate groups and residues Phe66 and Phe93 (Fig. 5C). In addition, the basestacking Phe141 in the cap enzyme is substituted with Arg158 in RsmG, which is likely to interfere with guanine binding in this orientation.

\section{Substrate preference for RsmG}

Our in vitro methylation experiments would seem to suggest that the preferred substrate for T. thermophilus RsmG is deproteinized $16 \mathrm{~S}$ rRNA. Both the preference for $16 \mathrm{~S}$ rRNA and the complete lack of activity at $10 \mathrm{mM} \mathrm{\textrm {Mg } ^ { 2 + }}$ indicate that the native $30 \mathrm{~S}$ subunit is unlikely to be the natural substrate in vivo. The most reasonable interpretation of these data is that the in vivo substrate is either nascent $16 \mathrm{~S}$ rRNA or an early subunit assembly intermediate. This behavior is distinct from that of the E. coli enzyme, which was reported to be unable to recognize naked 16S rRNA (Okamoto et al. 2007).

Inspection of the electrostatic surface of RsmG and of the location of the AMP molecule bound in data set RsmG2 shows a large, positively charged surface area that may represent a substrate interface region that recognizes $16 \mathrm{~S}$ rRNA (Fig. 5D). The additional C-terminal AMP-interacting region represents a significant part of this potential substratebinding interface, and these structural differences may result in a different mode of substrate binding between these two enzymes.

In any case, our results lead us to conclude that the intact 30 S subunit is very unlikely to be the natural substrate for T. thermophilus RsmG in vivo. This interpretation is consistent with the position of G527 in the intact 30S subunit (Wimberly et al. 2000), where it is buried and would be inaccessible for methylation without substantial unfolding of the local subunit structure. While deproteinized $16 \mathrm{~S}$ rRNA is the most active substrate in vitro, it should be remembered that, in vivo, several ribosomal proteins probably begin binding to the nascent $16 \mathrm{~S}$ rRNA transcript prior to its completion, making an early assembly intermediate a plausible candidate for the biological substrate of RsmG.

\section{MATERIALS AND METHODS}

\section{Growth and genetic manipulation of $T$. thermophilus}

T. thermophilus HB8 (ATCC27634) (Oshima and Imahori 1974) was grown aerobically at $72^{\circ} \mathrm{C}$ in ATCC medium 1598 (TEM) and on plates solidified with $2.8 \%$ Difco agar. Transformation of $T$. thermophilus HB8 with chromosomal or plasmid DNA was performed as described (Koyama et al. 1986; Hashimoto et al. 2001). 
Cloning was performed using standard methods. Restriction enzymes were purchased from Promega, Pfu DNA polymerase was purchased from Stratagene, AMV reverse transcriptase was purchased from Seikagaku, and oligonucleotides were purchased from Invitrogen.

\section{Construction of the $\Delta r s m G$ deletion allele}

A genomic sequence (935 base pairs [bp]) upstream of $r s m G$ and a genomic sequence (777 bp) downstream from $r s m G$ were amplified by PCR using oligonucleotide primer pairs Tth rsmG-9/Tth rsmG-10 and Tth rsmG8/Tth rsmG11, respectively (see below for primer sequences). These were inserted into KpnI--HindIII digested pUC18 as KpnI-PstI and PstI-HindIII fragments, respectively, to produce plasmid pUC18 $\Delta$ rsmG. The $h t k$ gene was then amplified from a pUC18 clone (a kind gift from H. Kagamiyama, Osaka Medical College) using primers HTK-18 and HTK-19 and inserted as a PstI restriction fragment into pUC18 $\Delta$ rsmG to generate pUC18 $\Delta \mathrm{rsmG}::$ htk. Constructs with $h t k$ in either orientation were obtained and were each introduced into T. thermophilus HB8 by natural transformation to kanamycin resistance. Transformants were purified by streaking for single colonies twice. Genomic DNA from positive clones was used to re-transform HB8 to kanamycin resistance, transformants were purified by streaking for single colonies twice, and the correct structure of the $r s m G$ regions of clones was verified by sequencing.

\section{Growth curves and growth competition assays}

Growth curves were established by diluting overnight cultures grown at $72^{\circ} \mathrm{C} 1: 100$ in TEM medium and culturing at $60^{\circ} \mathrm{C}$, $72^{\circ} \mathrm{C}$, or $80^{\circ} \mathrm{C}$. Growth was monitored by measuring turbidity at $600 \mathrm{~nm}$. Growth competition was performed by mixing equal volumes of overnight cultures of the two strains and growing for 24-h cycles. After each cycle, dilutions were plated on TEM plates and TEM plates containing $30 \mu \mathrm{g} / \mathrm{mL}$ kanamycin to monitor HB8 and HG 917 populations, respectively. Cultures were then subcultured by 1:2000 dilution in TEM.

\section{Oligonucleotide primers}

Oligonucleotide primers used in construction of the $\Delta r s m G:: h t k 2$ allele included Tth rsmG-9 (5'-GAGGTACCACCGAGGTCTACC TCCAGGGCTTCTC-3'), Tth rsmG-10 (5'-CCGCTGCAGGTGTT TCCCGTGAAACATCAGGCG-3'), Tth rsmG8 (5'-CGAGACCG CTAAGCTTCCTGGACACGG-3'), and Tth rsmG11 (5'-CACCT GCAGCGGCATCCGTTATGCTAAGGGCC- $\left.3^{\prime}\right)$; the $h t k$ gene was amplified for cloning using primers HTK-18 (5'-GAACTGCAGT ACCCGTTGACGGCGGATATGG-3') and HTK-19 (5' -GCTTGC ATGCCTGCAGCGTAACCAACATG-3') (restriction recognition sites are underlined). Primers used to amplify and sequence the $r s m G$ region included Tth rsmG1 (5'-GAAGGACCTCGAGGCCTTCCG CATCC- $\left.3^{\prime}\right)$ and Tth rsmG2 (5'-GGTTGATGGCCGTGGTGGTC TTGCC- $3^{\prime}$ ). Primer Tth 16S-rsmG1 (5'-AGTGAATCCGGGTAA CGC-3') was used for primer extension analysis.

\section{Preparation of ribosomes and ribosomal subunits and primer extension analysis of $\mathrm{m7G}$ modification}

$70 \mathrm{~S}$ ribosomes were prepared as described previously (Thompson and Dahlberg 2004) except that $\beta$-mercaptoethanol was omitted to avoid reduction of the disulfide bond of RsmG. 30S subunits were prepared by dissociation of $70 \mathrm{~S}$ ribosomes in $1 \mathrm{mM} \mathrm{MgCl}$ buffer followed by fractionation on $10 \%-35 \%$ sucrose gradients in an SW28 rotor at $18,000 \mathrm{rpm}$ for $18 \mathrm{~h}$. To prepare $16 \mathrm{~S} \mathrm{rRNA}, 30 \mathrm{~S}$ ribosomal subunits were precipitated by adding 0.1 vol of $3 \mathrm{M}$ $\mathrm{NaOAc}$ (pH 5.4) and 3 vol of $95 \%$ ethanol. After $2 \mathrm{~h}$ at $-20^{\circ} \mathrm{C}$, the sample was centrifuged and the pellet was resuspended with nuclease-free water. 16S rRNA was then extracted one time with buffer-saturated phenol and $0.1 \%$ SDS, one time with 1:1 phenolchloroform, $2 \times$ with chloroform, and precipitated. The rRNAcontaining pellet was dried and dissolved in nuclease-free water.

For primer extension analysis, ribosomes were precipitated with 95\% ethanol and phenol extracted as described by Merryman and Noller (1998). To induce strand scission at $\mathrm{m}^{7} \mathrm{G}$ residues, rRNA was treated with $\mathrm{NaBH}_{4}$ and aniline (Krol and Carbon 1989); reactions included dimethyl sulfate-methylated carrier tRNA as described (Zueva et al. 1985). Modified RNA was subjected to primer extension using AMV reverse transcriptase (Seikagaku) and a ${ }^{32} \mathrm{P}$-end-labeled oligonucleotide primer, Tth 16S-rsmG1, complementary to positions 565 to 548 of T. thermophilus $16 \mathrm{~S}$ rRNA. Extension products were resolved on $13 \%$ acrylamide $8 \mathrm{M}$ urea gels.

\section{In vitro methylation of $30 \mathrm{~S}$ ribosomal subunits and deproteinized 165 rRNA by RsmG}

Methylation reactions contained 150 pmol of $30 \mathrm{~S}$ ribosomal subunits from the $\Delta r s m G:: h t k 2$ mutant HG 917, $5 \mu \mathrm{L}$ of $\left[{ }^{3} \mathrm{H}\right]-$ $S$-adenosyl-L-methionine $(250 \mu \mathrm{M} ; 1000 \mathrm{cpm} / \mathrm{pmol})$ at $72^{\circ} \mathrm{C}$ in $150 \mu \mathrm{L}$ of a buffer containing $30 \mathrm{mM}$ HEPES-KOH ( $\mathrm{pH} 7.6$ ), $100 \mathrm{mM} \mathrm{NH}_{4} \mathrm{Cl}$, and $\mathrm{MgOAc}_{2}(1 \mathrm{mM}-10 \mathrm{mM})$. Reactions were initiated by addition of $30 \mathrm{pmol}$ of RsmG and incubated at $72^{\circ} \mathrm{C}$. At each time point, $20-\mu \mathrm{L}$ aliquots were removed, and the reactions were terminated by addition of $10 \%$ trichloroacetic acid (TCA). Samples were filtered through fiberglass filters (Whatman) and washed three times with $1 \mathrm{~mL}$ of 5\% TCA. Filters were dried and counted in a Beckman scintillation counter. In vitro methylation of $16 \mathrm{~S}$ rRNA was carried out at the same conditions used for $30 S$ subunits except for the incubation time, which was reduced since the reaction is faster than the methylation of intact 30 S subunits.

\section{Cloning, expression, and purification of RsmG}

The full-length native $r s m G$ gene (GenBank AP008226) from $T$. thermophilus HB8 genomic DNA was amplified by polymerase chain reaction (PCR) using a forward primer containing an NdeI restriction site (GGAATTCATATGTTTCACGGGAAACACCCGG GG) and a reverse primer containing an NotI restriction site (GGGTGCGGCCGCTTAGCATAACGGATGCCGCTCGGG). PCRs were performed using a touch-down protocol in the presence of $4 \%$ DMSO. The amplified $r s m G$ gene was inserted into the expression vector pET26b (Novagen) and transformed into the $E$. coli $\mathrm{DH} 5 \alpha$ cloning strain and plated onto LB-kanamycin plates. The final construct was introduced into an E. coli BL21 DE3 Star expression strain (Invitrogen).

Bacterial cells were grown to mid-log phase in $\mathrm{LB}$ media at $37^{\circ} \mathrm{C}$ in the presence of $35 \mu \mathrm{g} / \mathrm{mL}$ kanamycin. Protein expression was induced at $20^{\circ} \mathrm{C}$ by introduction of $400 \mu \mathrm{M}$ IPTG. Cells were pelleted after $18 \mathrm{~h}$ by centrifugation at $4000 \mathrm{rpm}$ for $20 \mathrm{~min}$ at $4^{\circ} \mathrm{C}$ and lysed by ultrasonification on ice in a buffer containing $20 \mathrm{mM}$ Tris-HCl (pH 6.8), 0.1\% Triton X-100, and 5\% glycerol. Cell 
debris and membranes were pelleted by centrifugation at 15,000 rpm for $30 \mathrm{~min}$ at $4^{\circ} \mathrm{C}$. The soluble fraction was heat-treated for $30 \mathrm{~min}$ at $65^{\circ} \mathrm{C}$ to denature and precipitate E. coli proteins, which were then separated by centrifugation at $15,000 \mathrm{rpm}$ for $30 \mathrm{~min}$ at $4^{\circ} \mathrm{C}$. The soluble native $T$. thermophilus $\mathrm{RsmG}$ was further purified by cation exchange chromatography (SP; GE Healthcare) at $\mathrm{pH} 6.8$, using a linear gradient of $10 \mathrm{mM}$ to $1 \mathrm{M} \mathrm{NaCl}$ concentration. RsmG fractions were concentrated and applied to a size-exclusion S200 column (GE Healthcare) equilibrated with buffer containing $20 \mathrm{mM}$ Tris- $\mathrm{HCl}$ (pH 6.8) and $200 \mathrm{mM} \mathrm{NaCl}$. The purified RsmG was buffer-exchanged into $10 \mathrm{mM}$ Tris- $\mathrm{HCl}$ ( $\mathrm{pH} 8.0$ ) and concentrated to $10 \mathrm{mg} / \mathrm{mL}$ for crystallization trials. To form the RsmG-AdoMet complex, purified RsmG was mixed with $4 \mathrm{mM}$ AdoMet and incubated for $2 \mathrm{~h}$ at $4^{\circ} \mathrm{C}$ before performing crystallization experiments. To form the RsmG-AdoMetAMP complex, purified RsmG was first mixed with $4 \mathrm{mM}$ AdoMet, and then $1 \mathrm{mM}$ AMP was added, incubated for $15 \mathrm{~min}$ at $65^{\circ} \mathrm{C}$, and slowly cooled to room temperature and filtered before performing the crystallization experiments. Crystals for the RsmG4 data set were obtained after purified protein was mixed with $1 \mathrm{mM}$ guanosine hydrate, incubated at $50^{\circ} \mathrm{C}$ for $10 \mathrm{~min}$, slowly cooled to room temperature, and filtered through a $0.2 \mu \mathrm{m}$ filter before crystallization.

\section{Crystallization}

All crystals were obtained using the microbatch technique under oil at $4^{\circ} \mathrm{C}$. To obtain the RsmG1 crystal form, $1 \mu \mathrm{L}$ of RsmGAdoMet complex solution was mixed with a reservoir solution containing 20\% (w/v) PEG1000, $100 \mathrm{mM}$ imidazole ( $\mathrm{pH} 8.0$ ), and $200 \mathrm{mM}$ calcium acetate. Initial crystals grew over the course of 2-3 mo with maximum dimensions of $0.1 \mathrm{~mm} \times 0.1 \mathrm{~mm} \times 0.3$ $\mathrm{mm}$. To obtain the RsmG2 crystal form, $1 \mu \mathrm{L}$ of RsmG-AdoMetAMP complex was mixed with the reservoir solution containing $10 \%$ (w/v) PEG8000, $100 \mathrm{mM}$ imidazole ( $\mathrm{pH}$ 8.0). Initial crystals grew over the course of 2-3 d with maximum dimensions of $0.3 \mathrm{~mm} \times 0.3 \mathrm{~mm} \times 0.4 \mathrm{~mm}$. To obtain the RsmG3 crystal form, $1 \mu \mathrm{L}$ of RsmG-AdoMet complex was mixed with the reservoir solution containing 20\% (w/v) PEG1000, $100 \mathrm{mM}$ imidazole ( $\mathrm{pH}$ 8.0 ), and $200 \mathrm{mM}$ calcium acetate. Initial crystals grew over the course of 2-3 mo with maximum dimensions of $0.15 \mathrm{~mm} \times 0.15$ $\mathrm{mm} \times 0.4 \mathrm{~mm}$. To obtain the RsmG4 crystal form, $1 \mu \mathrm{L}$ of RsmG-guanosine hydrate complex was mixed with the reservoir solution containing $30 \%(\mathrm{v} / \mathrm{v})$ methyl-2,4-pentanediol (MPD). Initial crystals grew over the course of $6-8 \mathrm{wk}$ with maximum dimensions of $0.2 \mathrm{~mm} \times 0.2 \mathrm{~mm} \times 0.4 \mathrm{~mm}$. RsmG1 and RsmG3 complex crystals were cryoprotected in mother liquor supplemented with 30\% PEG200 and then flash frozen by plunging into liquid nitrogen. RsmG2 complex crystals were cryoprotected in mother liquor supplemented with $20 \%$ ethylene glycol and then flash-frozen by plunging into liquid nitrogen. RsmG4 crystals were flash-frozen by plunging into liquid nitrogen directly from their mother liquor.

\section{Data collection}

X-ray diffraction data for RsmG1, RsmG2, and RsmG3 crystals were collected on a MAR CCD detector at the X4C beamline of the National Synchrotron Light Source in Brookhaven at a wavelength of $0.979 \AA$ and $-180^{\circ} \mathrm{C}$. Diffraction data for RsmG4 crystal were collected on an ADSC CCD detector at the X4A beamline of the National Synchrotron Light Source in Brookhaven at a wavelength of $0.979 \AA$ and $-180^{\circ} \mathrm{C}$. Diffraction data to $1.87 \AA$ for RsmG1 were collected in space group $\mathrm{P} 6_{1}$ with cell dimensions $a=77.8 \AA, b=77.8 \AA$, and $c=167.7 \AA$. Diffraction data for RsmG2 in space group $\mathrm{P}_{1}$ were collected to $1.5 \AA$ resolution with cell dimensions $a=77.2 \AA, b=77.2 \AA$, and $c=167.8 \AA$. Diffraction data to $2.1 \AA$ for RsmG3 were collected in space group $\mathrm{P}_{1}$ with cell dimensions $a=134.8 \AA$, $b=134.8 \AA$, and $c=167.1 \AA$. Diffraction data to $2.1 \AA$ for RsmG4 were collected in space group I222 with cell dimensions $a=110.1 \AA$, $b=114.0 \AA$, and $c=132.7 \AA$. A single crystal was used for each data set. Diffraction images were processed and scaled with the HKL2000 package (Otwinowski and Minor 1997). The data processing statistics are summarized in Table 2.

\section{Structure determination and refinement}

The RsmG1 structure was solved by molecular replacement with the program Phaser (McCoy et al. 2007). A search model was built with Modeller (Fiser and Sali 2003) based on the B. subtilis RsmG structure (PDB code 1XDZ; Midwest Center for Structural Genomics). Structure factors calculated after initial refinement with Refmac (Murshudov et al. 1997) from the CCP4 package (Bailey 1994) were used for subsequent model building with ARP/ wARP (Cohen et al. 2004). The resulting model was $90 \%$ complete and manually checked and completed with Coot (Emsley and Cowtan 2004). The atomic coordinates from the RsmG1 model were then used for initial refinement of the RsmG-AdoMet-AMP complex structure in space group $\mathrm{P}_{1}$ (RsmG2, $1.50 \AA$ A resolution). Structures of the AdoHcy-bound form in space group $\mathrm{P}_{1}$ (RsmG3, $2.1 \AA$ resolution) and of the second RsmG-AdoMet complex structure in space group I222 (RsmG4, $2.1 \AA$ resolution) were subsequently solved by molecular replacement using RsmG1 as search model. All models were evaluated with the MolProbity server (Davis et al. 2007), and TLS refinement was performed based on an analysis with the TLSMD server (Painter and Merritt 2006). Final crystallographic refinement steps were performed with Phenix (Adams et al. 2002). Residues in a flexible loop region between residues 46 and 57 were disordered and not included in the final models for data sets RsmG1-3. There are two molecules in the asymmetric unit in data sets RsmG1, RsmG2, and RsmG4, and six molecules in data set RsmG3. The crystallographic $R / R_{\text {free }}$ factors are $0.17 / 0.20,0.18 / 0.21,0.17 / 0.23$, and $0.18 / 0.20$ for data sets RsmG1, RsmG2, RsmG3, and RsmG4, respectively. The stereochemical quality of the models was assessed with Procheck (Laskowski et al. 1993). The Ramachandran statistics (most favored/additionally allowed/generously allowed/disallowed) are 96.6/3.4/0.0/0.0\% for RsmG1, 96.6/3.4/0.0/0.0\% for RsmG2, 96.6/ $3.4 / 0.0 / 0.0 \%$ for RsmG3, and 96.6/3.4/0.0/0.0\% for RsmG4. The refinement statistics are summarized in Table 2. Figures were generated using PyMOL (Delano Scientific), APBS (Baker et al. 2001), and JalView (Clamp et al. 2004). Sequence alignments were generated with ClustalW2 (Larkin et al. 2007), MultiProt (Shatsky et al. 2004), and Staccato (Shatsky et al. 2006).

\section{Accession codes}

Coordinates and structure factors have been deposited in the Protein Data Bank with accession codes 3G88, 3G89, 3G8A, and 3G8B for data sets RsmG1-4, respectively. 


\section{SUPPLEMENTAL MATERIAL}

Supplemental material can be found at http://www.rnajournal.org.

\section{ACKNOWLEDGMENTS}

We thank John Schwanof and Randy Abramowitz for access to the $\mathrm{X} 4 \mathrm{~A}$ and $\mathrm{X} 4 \mathrm{C}$ beamlines at the National Synchrotron Light Source, Megha Katti and Ashwin Cadambi for help with protein preparation, and $\mathrm{Hua} \mathrm{Li}$ for help with data collection at the synchrotron. This work was supported by grant GM19756 from the U.S. National Institutes of Health to A.E.D. and by Brown University to G.J.

Received March 17, 2009; accepted June 17, 2009.

\section{REFERENCES}

Adams PD, Grosse-Kunstleve RW, Hung LW, Ioerger TR, McCoy AJ, Moriarty NW, Read RJ, Sacchettini JC, Sauter NK, Terwilliger TC. 2002. PHENIX: Building new software for automated crystallographic structure determination. Acta Crystallogr D Biol Crystallogr 58: $1948-1954$.

Bailey S. 1994. The CCP4 Suite-programs for protein crystallography. Acta Crystallogr D Biol Crystallogr 50: 760-763.

Baker NA, Sept D, Joseph S, Holst MJ, McCammon JA. 2001. Electrostatics of nanosystems: Application to microtubules and the ribosome. Proc Natl Acad Sci 98: 10037-10041.

Beeby M, O'Connor BD, Ryttersgaard C, Boutz DR, Perry LJ, Yeates TO. 2005. The genomics of disulfide bonding and protein stabilization in thermophiles. PLoS Biol 3: e309. doi: 10.1371/ journal.pbio.0030309.

Carr JF, Gregory ST, Dahlberg AE. 2005. Severity of the streptomycin resistance and streptomycin dependence phenotypes of ribosomal protein S12 of Thermus thermophilus depends on the identity of highly conserved amino acid residues. J Bacteriol 187: 35483550 .

Carter AP, Clemons WM, Brodersen DE, Morgan-Warren RJ, Wimberly BT, Ramakrishnan V. 2000. Functional insights from the structure of the $30 \mathrm{~S}$ ribosomal subunit and its interactions with antibiotics. Nature 407: 340-348.

Chow CS, Lamichhane TN, Mahto SK. 2007. Expanding the nucleotide repertoire of the ribosome with post-transcriptional modifications. ACS Chem Biol 2: 610-619.

Clamp M, Cuff J, Searle SM, Barton GJ. 2004. The Jalview Java alignment editor. Bioinformatics 20: 426-427.

Cohen SX, Morris RJ, Fernandez FJ, Ben Jelloul M, Kakaris M, Parthasarathy V, Lamzin VS, Kleywegt GJ, Perrakis A. 2004. Toward complete validated models in the next generation of ARP/wARP. Acta Crystallogr D Biol Crystallogr 60: 2222-2229.

Davis IW, Leaver-Fay A, Chen VB, Block JN, Kapral GJ, Wang X, Murray LW, Arendall WB, Snoeyink J, Richardson JS, et al. 2007. MolProbity: All-atom contacts and structure validation for proteins and nucleic acids. Nucleic Acids Res 35: W375-W383.

Decatur WA, Fournier MJ. 2002. rRNA modifications and ribosome function. Trends Biochem Sci 27: 344-351.

Demirci H, Gregory ST, Dahlberg AE, Jogl G. 2007. Recognition of ribosomal protein $\mathrm{L} 11$ by the protein trimethyltransferase PrmA. EMBO J 26: 567-577.

Demirci H, Gregory ST, Dahlberg AE, Jogl G. 2008a. Crystal structure of the Thermus thermophilus $16 \mathrm{~S}$ rRNA methyltransferase RsmC in complex with cofactor and substrate guanosine. J Biol Chem 283: 26548-26556.

Demirci H, Gregory ST, Dahlberg AE, Jogl G. 2008b. Multiple-site trimethylation of ribosomal protein L11 by the PrmA methyltransferase. Structure 16: 1059-1066.
Demirci H, Belardinelli R, Seri E, Gregory ST, Gualerzi C, Dahlberg AE, Jogl G. 2009. Structural rearrangements in the active site of the Thermus thermophilus 16S rRNA methyltransferase KsgA in a binary complex with $5^{\prime}$-methylthioadenosine. J Mol Biol 388: 271-282.

Emsley P, Cowtan K. 2004. Coot: Model-building tools for molecular graphics. Acta Crystallogr D Biol Crystallogr 60: 2126-2132.

Fabrega C, Hausmann S, Shen V, Shuman S, Lima CD. 2004. Structure and mechanism of mRNA cap (guanine-N7) methyltransferase. Mol Cell 13: 77-89.

Fiser A, Sali A. 2003. Modeller: Generation and refinement of homology-based protein structure models. Methods Enzymol 374: 461-491.

Gregory ST, Dahlberg AE. 2008. Transposition of an insertion sequence, ISTth7, in the genome of the extreme thermophile Thermus thermophilus HB8. FEMS Microbiol Lett 289: 187-192.

Gregory ST, Dahlberg AE. 2009. Genetic and structural analysis of base substitutions in the central pseudoknot of Thermus thermophilus $16 \mathrm{~S}$ ribosomal RNA. RNA 15: 215-223.

Gregory ST, Cate JHD, Dahlberg AE. 2001. Streptomycin-resistant and streptomycin-dependent mutants of the extreme thermophile Thermus thermophilus. J Mol Biol 309: 333-338.

Gregory ST, Carr JF, Dahlberg AE. 2009. A signal relay between ribosomal protein $\mathrm{S} 12$ and elongation factor EF-Tu during decoding of mRNA. RNA 15: 208-214.

Gustilo EM, Vendeix FA, Agris PF. 2008. tRNA's modifications bring order to gene expression. Curr Opin Microbiol 11: 134-140.

Guymon R, Pomerantz SC, Crain PF, McCloskey JA. 2006. Influence of phylogeny on post-transcriptional modification of rRNA in thermophilic prokaryotes: The complete modification map of $16 \mathrm{~S}$ rRNA of Thermus thermophilus. Biochemistry 45: 48884899.

Hashimoto Y, Yano T, Kuramitsu S, Kagamiyama H. 2001. Disruption of Thermus thermophilus genes by homologous recombination using a thermostable kanamycin-resistant marker. FEBS Lett 506: 231-234.

Helser TL, Davies JE, Dahlberg JE. 1972. Mechanism of kasugamycin resistance in Escherichia coli. Nat New Biol 235: 6-9.

Holm L, Kaariainen S, Rosenstrom P, Schenkel A. 2008. Searching protein structure databases with DaliLite v.3. Bioinformatics 24: $2780-2781$.

Ireton K, Gunther NW, Grossman AD. 1994. spo0J is required for normal chromosome segregation as well as the initiation of sporulation in Bacillus subtilis. J Bacteriol 176: 5320-5329.

Johansen SK, Maus CE, Plikaytis BB, Douthwaite S. 2006. Capreomycin binds across the ribosomal subunit interface using tlyAencoded 2'-O-methylations in $16 \mathrm{~S}$ and $23 \mathrm{~S}$ rRNAs. Mol Cell 23: 173-182.

Kinghorn SM, O’Byrne CP, Booth IR, Stansfield I. 2002. Physiological analysis of the role of truB in Escherichia coli: A role for tRNA modification in extreme temperature resistance. Microbiology 148: 3511-3520.

Koyama Y, Hoshino T, Tomizuka N, Furukawa K. 1986. Genetic transformation of the extreme thermophile Thermus thermophilus and of other Thermus spp. J Bacteriol 166: 338-340.

Krol A, Carbon P. 1989. A guide for probing native small nuclear RNA and ribonucleoprotein structures. Methods Enzymol 180: 212-227.

Kurland CG, Hughes D, Ehrenberg M. 1996. Limitations of translational accuracy. In Escherichia coli and Salmonella: Cellular and molecular biology (eds. FC Neidhardt et al.), pp. 979-1004. American Society for Microbiology, Washington, DC.

Larkin MA, Blackshields G, Brown NP, Chenna R, McGettigan PA, McWilliam H, Valentin F, Wallace IM, Wilm A, Lopez R, et al. 2007. Clustal $\mathrm{W}$ and Clustal $\mathrm{X}$ version 2.0. Bioinformatics 23: 2947-2948.

Laskowski RA, MacArthur MW, Moss DS, Thornton JM. 1993. Procheck-a program to check the stereochemical quality of protein structures. J Appl Crystallogr 26: 283-291. 


\section{Gregory et al.}

Mallick P, Boutz DR, Eisenberg D, Yeates TO. 2002. Genomic evidence that the intracellular proteins of archaeal microbes contain disulfide bonds. Proc Natl Acad Sci 99: 9679-9684.

Marti-Renom MA, Stuart AC, Fiser A, Sanchez R, Melo F, Sali A. 2000. Comparative protein structure modeling of genes and genomes. Annu Rev Biophys Biomol Struct 29: 291-325.

McCoy AJ, Grosse-Kunstleve RW, Adams PD, Winn MD, Storoni LC, Read RJ. 2007. Phaser crystallographic software. J Appl Crystallogr 40: 658-674.

Merryman C, Noller HF. 1998. Footprinting and modificationinterference analysis of binding sites on RNA. In RNA:protein interactions. A practical approach (ed. CJW Smith), pp. 237-253. IRL Press, Oxford, UK.

Murray H, Errington J. 2008. Dynamic control of the DNA replication initiation protein DnaA by Soj/ParA. Cell 135: 74-84.

Murshudov GN, Vagin AA, Dodson EJ. 1997. Refinement of macromolecular structures by the maximum-likelihood method. Acta Crystallogr D Biol Crystallogr 53: 240-255.

Nishimura K, Hosaka T, Tokuyama S, Okamoto S, Ochi K. 2007a Mutations in $r s m G$, encoding a $16 \mathrm{~S}$ rRNA methyltransferase, result in low-level streptomycin resistance and antibiotic overproduction in Streptomyces coelicolor A3(2). J Bacteriol 189: 3876-3883.

Nishimura K, Johansen SK, Inaoka T, Hosaka T, Tokuyama $\mathrm{S}$, Tahara Y, Okamoto S, Kawamura F, Douthwaite S, Ochi K. 2007b. Identification of the RsmG methyltransferase target as $16 \mathrm{~S}$ rRNA nucleotide G527 and characterization of Bacillus subtilis rsmG mutants. J Bacteriol 189: 6068-6073.

Okamoto S, Tamaru A, Nakajima C, Nishimura K, Tanaka Y, Tokuyama S, Suzuki Y, Ochi K. 2007. Loss of a conserved 7-methylguanosine modification in 16S rRNA confers low-level streptomycin resistance in bacteria. Mol Microbiol 63: 1096-1106.

Oshima T, Imahori K. 1974. Description of Thermus thermophilus (Yoshida and Oshima), comb. nov., a nonsporulating thermo- philic bacterium from a Japanese thermal spa. Int J Syst Bacteriol 24: $102-112$.

Otwinowski Z, Minor W. 1997. Processing of X-ray diffraction data collected in oscillation mode. Methods Enzymol 276: 307326.

Painter J, Merritt EA. 2006. TLSMD web server for the generation of multi-group TLS models. J Appl Crystallogr 39: 109-111.

Peattie DA. 1979. Direct chemical method for sequencing RNA. Proc Natl Acad Sci 76: 1760-1764.

Romanowski MJ, Bonanno JB, Burley SK. 2002. Crystal structure of the Escherichia coli glucose-inhibited division protein B (GidB) reveals a methyltransferase fold. Proteins 47: 563-567.

Shatsky M, Nussinov R, Wolfson HJ. 2004. A method for simultaneous alignment of multiple protein structures. Proteins 56: 143156.

Shatsky M, Nussinov R, Wolfson HJ. 2006. Optimization of multiplesequence alignment based on multiple-structure alignment. Proteins 62: 209-217.

Shigi N, Sakaguchi Y, Suzuki T, Watanabe K. 2006. Identification of two tRNA thiolation genes required for cell growth at extremely high temperatures. J Biol Chem 281: 14296-14306.

Thompson J, Dahlberg AE. 2004. Testing the conservation of the translational machinery over evolution in diverse environments: Assaying Thermus thermophilus ribosomes and initiation factors in a coupled transcription-translation system from Escherichia coli. Nucleic Acids Res 32: 5954-5961.

Wimberly BT, Brodersen DE, Clemons WM, Morgan-Warren RJ, Carter AP, Vonrhein C, Hartsch T, Ramakrishnan V. 2000. Structure of the 30S ribosomal subunit. Nature 407: 327-339.

Zueva VS, Mankin AS, Bogdanov AA, Baratova LA. 1985. Specific fragmentation of tRNA and rRNA at a 7-methylguanine residue in the presence of methylated carrier RNA. Eur J Biochem 146: 679687. 

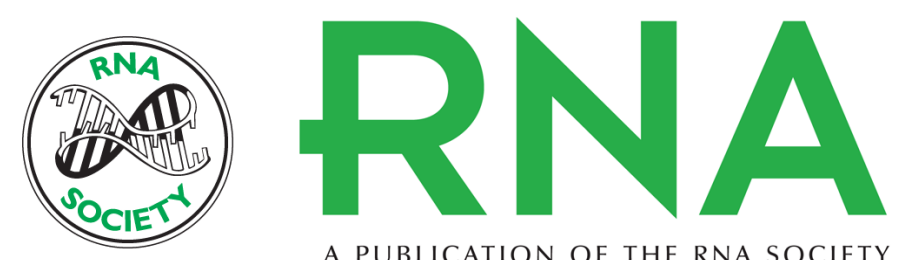

A PUBLICATION OF THE RNA SOCIETY

\section{Structural and functional studies of the Thermus thermophilus $16 \mathrm{~S}$ rRNA methyltransferase RsmG}

Steven T. Gregory, Hasan Demirci, Riccardo Belardinelli, et al.

RNA 2009 15: 1693-1704 originally published online July 21, 2009

Access the most recent version at doi:10.1261/rna.1652709

\section{Supplemental http://rnajournal.cshlp.org/content/suppl/2009/07/21/rna.1652709.DC1 \\ Material}

References This article cites 53 articles, 12 of which can be accessed free at: http://rnajournal.cshlp.org/content/15/9/1693.full.html\#ref-list-1

\section{License}

Email Alerting Receive free email alerts when new articles cite this article - sign up in the box at the Service top right corner of the article or click here. 